\title{
Osteoarthritis Prevention
}

\author{
Tuhina Neogi and Yuqing Zhang \\ Clinical Epidemiology Research and Training Unit, Boston University School of Medicine
}

\begin{abstract}
Purpose of review-We discuss herein the recent published epidemiologic data regarding risk factors for incident and progressive knee osteoarthritis $(\mathrm{OA})$, and related knee pain to identify targets for primary and secondary prevention. We also discuss the recently identified methodologic challenges to the study of knee OA, particularly for identifying risk factors for OA progression.

Recent findings-Recent epidemiologic studies of knee OA have confirmed that being overweight and obese, as well as biomechanical factors such as knee injuries, leg-length inequalities, and likely malalignment increase the risk for incident knee OA. Obesity also appears to play a role in accelerating OA worsening. However, with the exception of malalignment, no risk factors for knee OA progression have been identified. Novel approaches to the study of knee pain have demonstrated a strong association between structural abnormalities and presence of knee pain, contrary to the "so-called" structure-symptom discordance, as well as between fluctuations of knee pain with changes in specific structural lesions. A number of methodologic issues have been identified which may explain, in part, the difficulty in identifying risk factors for knee OA, particularly OA progression.
\end{abstract}

Summary-Few new risk factors for knee OA have been identified. Without such knowledge, prevention of $\mathrm{OA}$ remains challenging.

\section{Keywords}

incident radiographic knee osteoarthritis; progressive radiographic knee osteoarthritis; knee pain; risk factors

\section{Introduction}

We aim to review recent updates in the epidemiology of OA. We first review the descriptive epidemiology of OA generally. We then review the recently published literature, with particular attention to modifiable risk factors for knee OA. Specifically we evaluate the evidence for primary prevention of incident $\mathrm{OA}$ and secondary prevention of OA progression. Finally, we highlight methodologic challenges in the study of risk factors for OA.

\section{Epidemiology of OA}

$\mathrm{OA}$ is the most common form of arthritis. ${ }^{1}$ Although prevalence estimates vary, likely related to underlying differences in study samples and definitions of OA (e.g., clinical versus $\mathrm{x}$-ray confirmed), recent estimates indicate a rise in OA prevalence with 27 million

Corresponding author information: Dr. Tuhina Neogi, 650 Albany St, Clin Epi Unit, Suite X200, Boston, MA, 02118, Tel: 617-638-5180, tneogi@bu.edu.

The authors have no conflict of interest. 
US adults age 25 and older having clinical OA of either the hand, knee, or hip joint, an increase from 21 million in $1995 .{ }^{1}$ Such increases in OA prevalence are likely related to aging of the population and rising prevalence of obesity. Approximately $27 \%$ of adults age 26 or older have radiographic hand OA, and of adults age 45 or older, 19-28\% have knee OA and $27 \%$ have hip OA. Racial/ethnic differences in the prevalence of OA have been noted. African Americans overall as well as African American men had higher prevalence of radiographic hip OA than Caucasians (32.1\% vs. $26.6 \%$, and $32.2 \%$ vs. $23.8 \%$, respectively), while there was no difference between African American and Caucasian women $(40.3 \%$ vs. $39.4 \%){ }^{2}$ Additionally, specific individual radiographic features were noted to differ between African Americans and Whites. ${ }^{3,4}$

The knee is among the most commonly studied joint affected by OA. For the purposes of epidemiologic studies and clinical trials, tibiofemoral knee OA is defined by a standardized radiographic grading system on the Kellgren and Lawrence (KL) grading scale of $0-4$, with $\mathrm{KL}$ grade $\geq 2$ representing definite tibiofemoral OA. ${ }^{5}$ Whole knee OA can be defined by radiographic OA in the tibiofemoral and/or the patellofemoral joints. With the advent of magnetic resonance imaging (MRI), numerous joint structures previously not visualized on radiographs can be examined. To date, no MRI definition of OA has been established, although individual structural lesions on MRI are well-described, including cartilage lesions, osteophytes, bone marrow lesions, synovitis, effusion, and subchondral bone attrition. Symptomatic OA is defined as the presence of radiographic OA in combination with knee symptoms attributable to OA. Not all individuals with radiographic OA have concomitant symptoms. Thus, risk factors for structural disease and symptomatic disease may not be the same.

\section{Recent Insights into Risk Factors for Incident Radiographic Knee OA: Opportunities for Primary Prevention}

A number of risk factors have long been identified for incident OA, including age, female gender, and obesity. ${ }^{1,6-8} \mathrm{~A}$ recent meta-analysis found those who were obese or overweight to have 2.96 times the risk of incident knee OA compared with those who were normal weight $\left(95 \%\right.$ CI 2.56-3.43). ${ }^{7}$ Those that were overweight but not obese had 2.18 times the risk of developing knee OA compared with their normal weight counterparts(95\% CI 1.862.55). Further, risk of incident knee OA appears to increase with increasing BMI, regardless of knee alignment. ${ }^{9}$ Thus, the totality of evidence suggests that weight is an important modifiable risk factor for incident $\mathrm{OA}$.

Knee injury confers a 4-fold increased risk of knee OA. ${ }^{7}$ Apart from sports-related injuries, it is possible that repetitive use of a joint can increase the risk for OA. One potential mechanism may be through the association of certain occupational activities, which have been previously associated with knee OA, ${ }^{10}$ with meniscal tears. For example, floor layers were more likely to have degenerative meniscal tears than graphic designers without any knee demands. ${ }^{11}$ In knees without any cartilage lesions at baseline, meniscal damage increased the risk for developing knee OA over 30 months, with an OR of 10 (95\% CI 3.529). ${ }^{12}$ Although the prevalence of meniscal abnormalities increases as the radiographic severity of knee OA increases, ${ }^{13}$ surgical intervention has not been shown to reduce these risks. ${ }^{14}$ These studies support the importance of maintaining an intact meniscus to protect against development of OA, with the implication that avoidance of knee injuries, whether sports or occupationally related, would be a rational primary preventive strategy.

Knee alignment, both static and dynamic, has important implications for load distribution within the knee. There have been conflicting findings regarding the effects of alignment on incident $\mathrm{OA},{ }^{15}, 16$ and a best evidence synthesis concluded there was lack of sufficient 
evidence to draw a conclusion. ${ }^{17}$ It is possible that malalignment may be a reflection of the severity of the disease, with joint space loss due to cartilage and meniscal abnormalities, and bony contour alterations occurring as part of the OA disease process contributing to malalignment. However, more recently, Sharma et al found that varus malalignment increased the incidence of knee OA. ${ }^{18}$ No study to date has evaluated the effects of malalignment on incident OA among knees without any MRI-based lesions.

Leg-length inequality(LLI) is an easily modifiable abnormality that can also affect lower extremity biomechanics. Persons with LLI of at least $2 \mathrm{~cm}$ were almost twice as likely to have prevalent radiographic knee OA, but no such association was noted for incident knee OA. ${ }^{19,20}$ Similar findings were noted in another study, in which persons with LLI of at least $1 \mathrm{~cm}$ were more likely to have prevalent radiographic knee OA (OR 1.9, 95\% CI 1.5-2.4) in the shorter limb. ${ }^{21}$ Again, no association was noted for incident radiographic knee OA, although LLI was associated with incident symptomatic knee OA. Such discrepant findings were also noted for knee extensor strength, which was associated with incident symptomatic knee OA, but not with incident radiographic OA. ${ }^{22}$ It is possible that the difference may be related to the patellofemoral joint being included in the evaluation of incident symptomatic whole knee OA, but not included in the definition of incident radiographic tibiofemoral OA in both of these studies.

Factors with potentially systemic effects have also been associated with incident knee OA. Nevitt $e t$ al recently confirmed the previous observation that highersystemic bone mineral density was associated with an increased risk of incident OA. ${ }^{23}$ Those in the highest quartiles of femoral neck and whole body BMD both had 2.3 times higher odds of incident radiographic knee OA (95\% CI 1.2-4.5, and 1.3-4.3, respectively). Whether this relationship is related to factors contributing to bone remodeling or peak bone mass that may be genetically determined, ${ }^{24}$ or whether the higher systemic BMD represents higher BMI load over the years prior to OA onset, itself a strong risk factor for OA, is not clear. Insights from an animal ecology study highlight the importance of the systemic effects of nutrition in early life as a potential contributor to OA susceptibility. ${ }^{25}$ However the effects of readily modifiable dietary factors in humans have been inconclusive. Studies of the relationship between vitamin $\mathrm{D}$ and knee OA have been conflicting. ${ }^{26,27}$ Vitamin K, which has potential bone and cartilage effects, has been associated previously cross-sectionally with OA, and recently with incident radiographic knee OA and MRI-based cartilage lesions. ${ }^{28-31}$ However, further studies are required to clarify the association between such dietary factors and incident knee OA. C-reactive protein(CRP), another systemic factor, has been inconsistently associated with incident knee OA. A recent study found no association between CRP and incident knee OA, largely explained by the confounding effects of BMI. ${ }^{32}$ Additionally, CRP genetic variation was not associated with incident knee OA.

Although genetic studies generally focus on prevalent knee OA, they do provide some insight into potential pathophysiologic pathways that can contribute to knee OA risk, and provide potentially rational targets for future therapeutic strategies. The gene for GDF5 has been associated with OA in recent studies, which have also identified LRCH1 and FRZB as additional promising candidates. ${ }^{33-35} \mathrm{~A}$ locus on chromosome $7 \mathrm{q} 22$ has also been recently associated with knee OA. ${ }^{36}$ Further work, particularly with regards to mechanistic studies, is needed in this area.

\section{Recent Insights into Risk Factors for Radiographic OA Progression: Opportunities for Secondary Prevention}

In contrast to incident radiographic knee $\mathrm{OA}$, few risk factors for knee OA progression have been identified. ${ }^{37}$ 
Most risk factors for incident knee OA, particularly systemic factors, have not been associated with progressive OA. For example, higher BMD was associated with incident knee OA, but it was not associated with progressive OA in the same study. ${ }^{23} \mathrm{Niu}$ et al has shown that high BMI was not associated with progressive radiographic knee OA, although it increased the risk of incident knee OA, and its effect seemed to be modified by malalignment. ${ }^{9}$ However, using the same data, Zhang et al demonstrated that high BMI increased the risk of both mild radiographic $\mathrm{OA}(\mathrm{KL}=2)$ and moderate-severe radiographic $\mathrm{OA}(\mathrm{KL}=3$ or 4$)$ among knees that were $\mathrm{KL}=0$ at baseline, respectively. ${ }^{38}$ Since knees that develop $\mathrm{KL}=3$ or 4 over time must have gone through the $\mathrm{KL}=2$ stage, this approach provides indirect evidence that obesity not only increases risk of incident $\mathrm{OA}$, but it also accelerates OA progression. A randomized controlled trial was recently conducted to evaluate vitamin D's effects, but did not demonstrate a beneficial effect on cartilage loss by MRI. ${ }^{39}$

Knee malalignment is one of the strongest predictors for progressive knee OA. ${ }^{18}$ However, no study to date has documented slowing of disease progression if alignment is corrected. Interestingly, in a randomized controlled trial of doxycycline versus placebo, varus malalignment was found to negate the potential chondroprotective effects of doxycycline. ${ }^{40}$

\section{Recent insights into risk factors for knee pain}

The study of knee pain is challenging. Clinically, symptoms related to knee OA are known to be activity-related in early stages, progressing to more persistent symptoms in late stages of disease that are punctuated with intermittent increased pain. ${ }^{41}$ In the Multicenter Osteoarthritis Study, approximately $40 \%$ of individuals had inconsistently frequent knee pain (that is, they stated they had frequent knee pain during only one of the two occasions they were asked on average 30 days apart). Such individuals had less severe radiographic $\mathrm{OA}$, less depressive symptoms, and less widespread pain. ${ }^{42} \mathrm{~A}$ recent study in which pain assessments were conducted weekly over 12 weeks, psychological factors fluctuated with pain severity, ${ }^{43}$ supporting an important link between the pain experience and psychological state. Indeed, because numerous factors, many of which are unmeasurable, can contribute to the pain experience, such as genetics, sociocultural environment, medications, among others in addition to psychological factors, a so-called "structure-symptom" discordance is often described in OA.

However, when such between-person variability or confounding factors are eliminated by using a within-person knee-matched study design(in which one knee has pain while the other does not), a strong association between radiographic severity and presence of frequent knee pain, consistent knee pain, and severity of knee pain can be discerned, even at the earliest stages of radiographic knee OA (Figure 1). ${ }^{44}$ Such findings indicate that certain structural lesions within the knee may be a cause of knee pain. Further, specific MRI features of OA that can change over time, such as bone marrow lesions, synovitis, and effusion, were associated with pain fluctuation. ${ }^{45}$ As these structural lesions worsened, so too did the likelihood that the knee would be painful. Similarly, as these abnormalities diminished in a knee, so too did the likelihood that the knee's pain had subsided. A recent systematic review indicated that the bulk of the evidence favors an association of BMLs and synovitis with the pain experience of $\mathrm{OA},{ }^{46}$ supporting further study into these abnormalities for rational therapeutic targeting. 


\section{Methodologic Challenges in the Study of Incident and Progressive Radiographic Knee OA}

Eligible knees for observational studies of incident radiographic OA are those that are either $\mathrm{KL}=0$ or $\mathrm{KL}=1$, a mixture of "pre-radiographic $\mathrm{OA}$ " $(\mathrm{KL}=1)$ and no early evidence of radiographic changes $(K L=0)$. A knee that starts at $K L=1$ can more readily achieve $K L \geq 2$ than a knee that starts at $\mathrm{KL}=0$. If the distribution of $\mathrm{KL}=0$ and $\mathrm{KL}=1$ varies by categories of the risk factor being studied, it could lead to potential bias. However, such an issue is avoided in a clinical trial by randomization. Furthermore, most risk factors for OA are chronic factors, such as obesity, leg-length inequality, or BMD. These chronic factors are likely to be present long before subjects are enrolled into a study. If those chronic risk factors have already caused a substantial proportion of subjects to develop knee OA, then it is quite possible that participants who are still exposed to such a risk factor without yet having developed OA would be less susceptible to knee OA compared with individuals who are not exposed to such a risk factor. For example, long-standing exposures such as obesity may have caused OA at an earlier age than those being studied, particularly in studies of older adults, but that true effect cannot be discerned since those individuals who already have knee OA are excluded from studies of incident disease. In contrast, individuals who have been obese for a long time and who are free of OA at the study onset may in fact be less susceptible to developing OA. Thus observational studies evaluating the association between a chronic exposure and incident knee OA may not be able to detect the true magnitude of effect. A strategy to overcome this problem is to study individuals earlier in the course of their exposure to such risk factors.

Some risk factors associated with incident disease are not associated with or are even paradoxically protective against progressive OA. In observational studies of OA progression, eligible knees consist of those that already have knee $\mathrm{OA}$, i.e., $\mathrm{KL}=2$ or $\mathrm{KL}=3$. Here again, the knees included at baseline are a "mixture" of differing degrees of severity that may vary among exposed and non-exposed groups. Furthermore, the outcome is also heterogeneous: knees that progress from $\mathrm{KL}=3$ to $\mathrm{KL}=4$ are considered the same as those that progress from $\mathrm{KL}=2$ to $\mathrm{KL}=3$ or to $\mathrm{KL}=4$. Thus $\mathrm{KL}=3$ knees are eligible for study entry, but $\mathrm{KL}=3$ also defines a study endpoint. Finally, studies of OA progression are, in essence, conducted to assess an association between a risk factor that causes OA initiation to progress to more severe OA. This results in conditioning on an "intermediate" stage of OA when assembling the study sample, i.e., by limiting the study sample to those who had mild to moderate knee OA at baseline, which can substantially dilute any potential effects of a risk factor on the risk of OA progression. ${ }^{38}$

\section{Conclusions}

OA is a common disease, contributing importantly to functional limitations and disability, particularly in older adults. Despite this, we know little about its risk factors. Methodologic challenges have hindered our ability to understand disease etiology, particularly for OA progression and symptomatic disease. Therefore prevention of OA remains challenging. Modifiable risk factors that have sufficient evidence to support intervention at the present time appears to primarily be that of weight loss and avoiding knee injury. This review of the recent literature supports the need for ongoing epidemiologic and intervention studies for the prevention of incident and progressive knee OA, as well as knee pain related to OA, with an eye to adopting novel approaches to circumvent some of the methodologic challenges identified. 


\title{
Acknowledgments
}

\author{
Funding: \\ Dr. Neogi is supported by:
}

NIAMS K23AR055127; Arthritis Foundation Arthritis Investigator Award; Boston Claude D. Pepper Older Americans Independence Center (P30-AG031679)

This work was additionally supported by:

NIH AR47785

\section{References}

1. Lawrence RC, Felson DT, Helmick CG, et al. Estimates of the prevalence of arthritis and other rheumatic conditions in the United States. Part II. Arthritis Rheum. 2008; 58:26-35. [PubMed: 18163497]

2. Jordan JM, Helmick CG, Renner JB, et al. Prevalence of hip symptoms and radiographic and symptomatic hip osteoarthritis in African Americans and Caucasians: the Johnston County Osteoarthritis Project. J Rheumatol. 2009; 36:809-15. [PubMed: 19286855]

3. Braga L, Renner JB, Schwartz TA, et al. Differences in radiographic features of knee osteoarthritis in African-Americans and Caucasians: the Johnston county osteoarthritis project. Osteoarthritis Cartilage. 2009; 17:1554-61. [PubMed: 19735758]

4. Nelson AE, Braga L, Renner JB, et al. Characterization of individual radiographic features of hip osteoarthritis in African American and White women and men: the Johnston County Osteoarthritis Project. Arthritis Care Res (Hoboken). 2010; 62:190-7. [PubMed: 20191517]

5. Kellgren, JH.; Lawrence, JS. Atlas of standard radiographs. Oxford (UK): Oxford University Press; 1963.

6. Felson DT, Anderson JJ, Naimark A, Walker AM, Meenan RF. Obesity and knee osteoarthritis. The Framingham Study. Ann Intern Med. 1988; 109:18-24. [PubMed: 3377350]

7*. Blagojevic M, Jinks C, Jeffery A, Jordan KP. Risk factors for onset of osteoarthritis of the knee in older adults: a systematic review and meta-analysis. Osteoarthritis Cartilage. 2010; 18:24-33. Meta-analysis of risk factors for incident knee OA. [PubMed: 19751691]

8. Srikanth VK, Fryer JL, Zhai G, Winzenberg TM, Hosmer D, Jones G. A meta-analysis of sex differences prevalence, incidence and severity of osteoarthritis. Osteoarthritis Cartilage. 2005; 13:769-81. [PubMed: 15978850]

9*. Niu J, Zhang YQ, Torner J, et al. Is obesity a risk factor for progressive radiographic knee osteoarthritis? Arthritis Rheum. 2009; 61:329-35. This study demonstrates the potential paradoxical effects of obesity as a risk factor for incident knee OA, but not for progressive knee OA. It also demonstrates that modification by alignment may explain the lack of association between obesity and progressive knee OA. [PubMed: 19248122]

10. Felson DT, Hannan MT, Naimark A, et al. Occupational physical demands, knee bending, and knee osteoarthritis: results from the Framingham Study. J Rheumatol. 1991; 18:1587-92. [PubMed: 1765986]

11. Rytter S, Egund N, Jensen LK, Bonde JP. Occupational kneeling and radiographic tibiofemoral and patellofemoral osteoarthritis. J Occup Med Toxicol. 2009; 4:19. [PubMed: 19594940]

12. Englund M, Guermazi A, Roemer FW, et al. Meniscal tear in knees without surgery and the development of radiographic osteoarthritis among middle-aged and elderly persons: The Multicenter Osteoarthritis Study. Arthritis Rheum. 2009; 60:831-9. [PubMed: 19248082]

13. Englund M, Guermazi A, Gale D, et al. Incidental meniscal findings on knee MRI in middle-aged and elderly persons. N Engl J Med. 2008; 359:1108-15. [PubMed: 18784100]

14. Lohmander LS, Englund PM, Dahl LL, Roos EM. The long-term consequence of anterior cruciate ligament and meniscus injuries: osteoarthritis. Am J Sports Med. 2007; 35:1756-69. [PubMed: 17761605] 
15. Brouwer GM, van Tol AW, Bergink AP, et al. Association between valgus and varus alignment and the development and progression of radiographic osteoarthritis of the knee. Arthritis Rheum. 2007; 56:1204-11. [PubMed: 17393449]

16. Hunter DJ, Niu J, Felson DT, et al. Knee alignment does not predict incident osteoarthritis: the Framingham osteoarthritis study. Arthritis Rheum. 2007; 56:1212-8. [PubMed: 17393450]

17. Tanamas S, Hanna FS, Cicuttini FM, Wluka AE, Berry P, Urquhart DM. Does knee malalignment increase the risk of development and progression of knee osteoarthritis? A systematic review. Arthritis Rheum. 2009; 61:459-67. [PubMed: 19333985]

18. Sharma L, Song J, Dunlop D, et al. Varus and valgus alignment and incident and progressive knee osteoarthritis. Ann Rheum Dis. 2010 Published online first 2010 June 1. 10.1136/ard.2010.129742

19*. Golightly YM, Allen KD, Helmick CG, Schwartz TA, Renner JB, Jordan JM. Hazard of Incident and Progressive Knee and Hip Radiographic Osteoarthritis and Chronic Joint Symptoms in Individuals with and without Limb Length Inequality. J Rheumatol. 2010; 37:2133-40. This is one of only two studies which has evaluated limb length inequality as a risk factor for incident knee OA. [PubMed: 20634243]

20. Golightly YM, Allen KD, Renner JB, Helmick CG, Salazar A, Jordan JM. Relationship of limb length inequality with radiographic knee and hip osteoarthritis. Osteoarthritis Cartilage. 2007; 15:824-9. [PubMed: 17321169]

21*. Harvey WF, Yang M, Cooke TD, et al. Association of leg-length inequality with knee osteoarthritis: a cohort study. Ann Intern Med. 2010; 152:287-95. This is one of only two studies which has evaluated leg-length inequality as a risk factor for incident knee OA. [PubMed: 20194234]

22. Segal NA, Torner JC, Felson D, et al. Effect of thigh strength on incident radiographic and symptomatic knee osteoarthritis in a longitudinal cohort. Arthritis Rheum. 2009; 61:1210-7. [PubMed: 19714608]

23*. Nevitt MC, Zhang Y, Javaid MK, et al. High systemic bone mineral density increases the risk of incident knee $\mathrm{OA}$ and joint space narrowing, but not radiographic progression of existing knee OA: the MOST study. Ann Rheum Dis. 2010; 69:163-8. This study demonstrates the paradoxical effect of BMD being associated with incident knee OA, but having no association with progressive knee OA. [PubMed: 19147619]

24. Naganathan V, Zochling J, March L, Sambrook PN. Peak bone mass is increased in the hip in daughters of women with osteoarthritis. Bone. 2002; 30:287-92. [PubMed: 11792599]

25*. Peterson RO, Vucetich JA, Fenton G, Drummer TD, Larsen CS. Ecology of arthritis. Ecol Lett. $2010 ; 13: 1124-8$. This study demonstrates the potential importance of early life nutrition on risk of OA. The implication for studying OA in humans is that dietary factors from earlier in life than older adulthood may be of most biologic relevance. [PubMed: 20618843]

26. Felson DT, Niu J, Clancy M, et al. Low levels of vitamin D and worsening of knee osteoarthritis: results of two longitudinal studies. Arthritis Rheum. 2007; 56:129-36. [PubMed: 17195215]

27. McAlindon T, Felson DT. Nutrition: risk factors for osteoarthritis. Ann Rheum Dis. 1997; 56:397400. [PubMed: 9485998]

28. Neogi T, Booth SL, Zhang YQ, et al. Low vitamin K status is associated with osteoarthritis in the hand and knee. Arthritis Rheum. 2006; 54:1255-61. [PubMed: 16572460]

29. Misra D, Booth SL, Felson D, et al. Vitamin K deficiency is associated with incident knee osteoarthritis and cartilage lesions on MRI: The MOST Study. Osteoarthritis Cartilage. 2010; 18:S28-S9. [PubMed: 20399895]

30. Neogi T, Felson DT, Sarno R, Booth SL. Vitamin K in hand osteoarthritis: results from a randomised clinical trial. Ann Rheum Dis. 2008; 67:1570-3. [PubMed: 18625626]

31. Oka H, Akune T, Muraki S, et al. Association of low dietary vitamin K intake with radiographic knee osteoarthritis in the Japanese elderly population: dietary survey in a population-based cohort of the ROAD study. J Orthop Sci. 2009; 14:687-92. [PubMed: 19997813]

32. Kerkhof HJ, Bierma-Zeinstra SM, Castano-Betancourt MC, et al. Serum C reactive protein levels and genetic variation in the CRP gene are not associated with the prevalence, incidence or progression of osteoarthritis independent of body mass index. Ann Rheum Dis. 2010 Published online first 2010 June 01. 10.1136/ard.2009.125260 
33. Evangelou E, Chapman K, Meulenbelt I, et al. Large-scale analysis of association between GDF5 and FRZB variants and osteoarthritis of the hip, knee, and hand. Arthritis Rheum. 2009; 60:1710 21. [PubMed: 19479880]

34. Valdes AM, Evangelou E, Kerkhof HJ, et al. The GDF5 rs143383 polymorphism is associated with osteoarthritis of the knee with genome-wide statistical significance. Ann Rheum Dis 2010. Published online first 2010 Sep 28. 10.1136/ard.2010.134155

35. Zintzaras E, Kitsios GD, Ziogas DC, Rodopoulou P, Karachalios T. Field synopsis and synthesis of genetic association studies in osteoarthritis: the CUMAGAS-OSTEO information system. Am J Epidemiol. 2010; 171:851-8. [PubMed: 20237151]

36. Kerkhof HJ, Lories RJ, Meulenbelt I, et al. A genome-wide association study identifies an osteoarthritis susceptibility locus on chromosome 7q22. Arthritis Rheum. 2010; 62:499-510. [PubMed: 20112360]

37. Belo JN, Berger MY, Reijman M, Koes BW, Bierma-Zeinstra SM. Prognostic factors of progression of osteoarthritis of the knee: a systematic review of observational studies. Arthritis Rheum. 2007; 57:13-26. [PubMed: 17266080]

38**. Zhang Y, Niu J, Felson DT, Choi HK, Nevitt M, Neogi T. Methodological challenges in studying risk factors for progression of knee osteoarthritis. Arthritis Care Res (Hoboken). 2010 Published online first 2010 Jul 10. This paper reviews the methodologic challenges in the study of progressive knee $\mathrm{OA}$, with specific exploration of biases resulting from study design and measurement issues, as well as potential alternative approaches to overcome such biases. 10.1002/acr.20287

39. McAlindon T, Dawson-Huges B, Driban J, et al. Clinical trial of vitamin D to reduce pain and structural progression of knee osteoarthritis (OA). Arthritis Rheum. 2010:62. In press.

40*. Mazzuca SA, Brandt KD, Chakr R, Lane KA. Varus malalignment negates the structuremodifying benefits of doxycycline in obese women with knee osteoarthritis. Osteoarthritis Cartilage. 2010; 18:1008-11. This paper explores the potential for biomechanical factors, malalignment inthis case, to affect the ability for an intervention's chondroprotective effects to be detected. [PubMed: 20493957]

41. Hawker GA, Stewart L, French MR, et al. Understanding the pain experience in hip and knee osteoarthritis--an OARSI/OMERACT initiative. Osteoarthritis Cartilage. 2008; 16:415-22. [PubMed: 18296075]

42. Neogi T, Nevitt MC, Yang M, Curtis JR, Torner J, Felson DT. Consistency of knee pain: correlates and association with function. Osteoarthritis Cartilage. 2010; 18:1250-5. [PubMed: 20708003]

43. Wise BL, Niu J, Zhang Y, et al. Psychological factors and their relation to osteoarthritis pain. Osteoarthritis Cartilage. 2010; 18:883-7. [PubMed: 20346403]

44**. Neogi T, Felson D, Niu J, et al. Association between radiographic features of knee osteoarthritis and pain: results from two cohort studies. BMJ. 2009; 339:b2844. This paper uses a novel methodology to demonstrate that, when between-person confounding is eliminated, there is a strong association between the structural changes of knee OA and presence of knee pain symptoms. Thus, the so-called "symptom-structure discordance" can be largely attributed to between-person variability in the pain experience. [PubMed: 19700505]

$45^{* *}$. Zhang YQ, Nevitt M, Niu JB, et al. Fluctuation of Knee Pain and Changes in Bone Marrow Lesions, Effusions and Synovitis on MRI: The MOST Study. Arthritis Rheum. In press. This paper uses a novel methodology to demonstrate that changes in MRI features of OA over time are associated with fluctuation in knee pain. Using a knee-matched approach, as a given knee's bone marrow lesions, synovitis, or effusion worsened or increased, so too did the risk of knee pain, and as such lesions improved or decreased, the risk of knee pain also decreased. These findings suggest that specific structures within the knee can contribute to knee pain.

46. Yusuf E, Kortekaas MC, Watt I, Huizinga TW, Kloppenburg M. Do knee abnormalities visualised on MRI explain knee pain in knee osteoarthritis? A systematic review. Ann Rheum Dis. 2010 Published online first 2010 Sep 09. 10.1135/ard.2010.131904 
Key Points

- Few risk factors for incident knee OA have been identified, and even fewer for progressive knee OA have been identified

- Structural joint pathology contributes to knee pain and its fluctuation

- Methodologic challenges have affected our ability to study OA progression and symptomatic disease

- There is a need for ongoing epidemiologic and intervention studies for the prevention of incident and progressive knee $\mathrm{OA}$ and related knee pain 


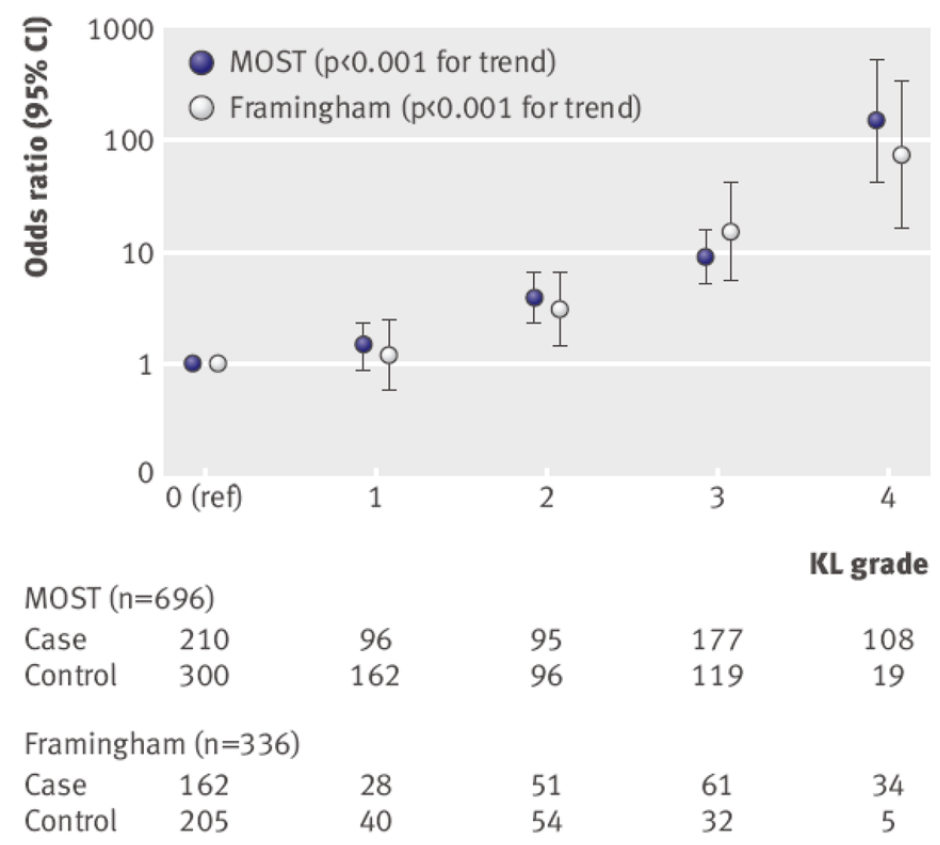

Figure 1.

Associations of frequent knee pain with Kellgren and Lawrence grade among individuals with two knees discordant for frequent knee pain status. The number of case knees(those with frequent knee pain) and control knees (those without frequent knee pain) are shown beneath the graph for each Kellgren and Lawrence grade. Note that the Y-axis is logarithmically scaled. Reprinted with permission from: Neogi, T Felson, D and Niu, J., et al. Association between radiographic features of knee osteoarthritis and pain: results from two cohort studies. 2009 BMJ 339:doi:10.1136/bmj.b2844. 